\title{
Custom Prosthetic Cover Development Methodology
}

\author{
Branko ŠTEFANOVIČ, Romana UHRÍNOVÁ, Monika MICHALÍKOVÁ, \\ Lucia BEDNARČíKOVÁ, Jozef ŽIVČÁK \\ Technical University of Košice, Faculty of Mechanical Engineering, \\ Department of Biomedical Engineering and Measurement, Košice, Slovakia
}

https://doi.org/10.15221/21.17

\begin{abstract}
A prosthetic covers function is to protect other components of the prosthesis and to increase its service life, while also fulfilling its aesthetic function. Its primary purpose is to prevent damage to the functional parts of the prosthesis and to prevent the penetration of various impurities into these parts of the prosthesis. Its aesthetic function is no less important for the user because it increases his mental comfort, socialization and allows the user to adjust his prosthesis in a unique way.

For amputees, the aesthetic aspect of their lost limb is often important. Dissatisfaction with their appearance leads to loss of self-esteem and depression, which can cause dissatisfaction in people's quality of life and can even lead to the complete rejection or non-use of the prosthesis. To improve these requirements, a proposal was made to develop a custom lower limb prosthetic cover.

Currently, there are companies that provide designer covers made using AM (additive manufacturing) or plastic moulding. The disadvantage, however, is their high price. For this reason, a methodology for the individual lower limb prosthesis cover design is proposed. The main aim of this new design is to be financially inexpensive and suitable for mass production.

In the first step, a database of foreleg 3D scans, with adequate circumferential and length measurements has been created. The database consists of 17 scanned subjects, 11 female and 6 males from the age range of 18 to 70 years. Total of 34 forelegs (left and right) scans have been acquired. The emphasis during scanning was on the target placement and positioning of the foreleg. The second step was the cover size categories development. In this step, the design of sizes for serial production, sorting, and arrangement of measured values into groups was made. The classification was performed based on the measured values. The last step was the custom cover design process. This process consisted of CAD software design based on the data acquired from the 3D scanner and measurement gauges.

The result of this study is a methodology for the correct selection from the appropriate prosthetic cover size category. Based on the foreleg database creation, the cover sizes have been divided into 3 categories (small, medium, large). All models have been designed for simple AM production. In the future, correct material and AM technology selection will be performed.
\end{abstract}

Key words: Lower limb, foreleg, prosthetic cover, CAD/CAM, 3D scanning.

\section{Introduction}

Currently, with the trend of an aging population, the proportion of the most common diseases of civilization is increasing, such as cardiovascular diseases and diabetes mellitus [1,2], the fatal consequences of which are often amputations of the lower limbs (more than $50 \%$ of the population suffering from these diseases). Also, car accidents and other traumatic events (1.5-2\% of all amputations [3], as well as various cancers, lead to irreversible surgery (amputation), to save human life. This handicap is solved by a prosthesis of the lower limb, which nowadays also meets the demanding requirements of the user. However, in the development, design and production, the focus is placed on functional aspect and often aesthetics is not fully considered. The aesthetic function of the prosthetic device is no less important for the user because it increases his mental comfort, socialization and allows him to uniquely adapt the prosthesis to his individual needs. Thus, aesthetic deficiency can have a strong psychological and cognitive effect, which will ultimately affect the usage, acceptance, or rejection of the prosthesis, as well as psychosocial well-being and user satisfaction.

There is a close link between physical appearance and user satisfaction [4]. Up to 93\% of users use prostheses daily (average of 12 hours) and up to $40 \%$ of users state appearance as a very important parameter, so it is important to combine aesthetics and functionality to provide psychological comfort and sense of style. The aesthetics of the shape and form can vary depending on gender, age, and lifestyle $[5,6,7]$. 
A prosthetic cover is therefore a suitable solution either due to the protective function of the prosthesis components, but mainly due to the prolongation of the prosthesis life against various external influences and aesthetic function fulfilment. The use of 3D scanning for the healthy limb shape acquisition and additive manufacturing technology for prosthetic cover production of lower limb prostheses offer many benefits, whether it is customer comfort, time savings, home comfort (no need to visit the orthotists workplace), various product design options (from very complicated shapes and patterns, up to the possibility of an exact shape replica of the preserved limb) and others.

There are several companies on the world market that are dedicated to the design and production of these devices (mostly mass production of standardized types of covers) which differ from each other by the material they use, the construction, type of clamping, external appearance, and colour. Companies such as UNYQ (San Francisco, USA), Alleles Design Studio (Victoria, British Columbia, Canada), Art4Leg (Prague, Czech Republic), and Limb-Art (Conwy, Great Britain) have been providing affordable, personalized lower-prosthetic fairings via 3D-printing technologies for more than a half-dozen years, which are made from ABS (Acrylonitrile butadiene styrene), PA (Polyamide), nylon, and other materials [8-11]. The disadvantage of these companies is the absence of covers of anatomical natural appearance and, of course, high financial costs, which range from 250-1500€ depending on the material, construction, method of production, design, patterns, and finishes. Another disadvantage of these devices is that they are not reimbursed by the health insurance company because they are not necessary for the functionality of the prosthesis, only the covers that are part of the prosthesis are reimbursed.

The aim of this work is to create a prosthetic cover for a transtibial prosthesis with the greatest possible degree of personalization and minimization of production costs. These requirements will be ensured by the creation of a database of scans of the preserved limb obtained using the Artec Eva 3D scanner. The creation of a database for the design of a prosthetic cover model will be based on the shape of the foreleg, which is generalizable and can be scaled according to selected dimensional parameters (length, circumference), while understanding individual shape deviations between the target group. This can be achieved by developing a database that contains large samples of anthropometric data based on the age and gender of end users. Subsequently, an average model of the foreleg area in several size variants is created as a basis for the prosthetic cover model, which is manufactured using a low-cost 3D printer.

\section{Method}

The content of this chapter is the creation of a database of calf scans, for the purposes of serial production of prosthetic covers for below knee prosthesis. A set of 17 subjects was organised for this purpose. This group included 11 women and 6 men between the ages of 18 and 70 . The acquisition of specific data is the most important part in the production of prosthetic coverings, due to the correct design of the size and achieving symmetry with a healthy limb. For the purposes of this work, we chose the following procedure, in the first phase a manual sampling was performed by a band gauge and in the second phase, 3D scanning was performed using an optical scanner Artec Eva (Artec 3D, Luxembourg, Luxembourg).

\subsection{Measurement}

To minimize measurement errors and to avoid inaccuracies due to incorrectly chosen place on the forelegs, 3 basic anthropometric points and axes of the knee joint were selected during the measurement, which must be marked when taking circumferential and length parameters (Fig. 1). These dots are marked with coloured stickers on the forelegs to highlight them for scanning.

These points were chosen to determine the required circumferential and length measurements such as the circumference below the knee, which is important for the correct adhesion of the cover to the prosthetic socket, the minimum circumference above the ankle required to properly fit the cover to the prosthetic foot and the maximum length from the knee joint axis to the outer ankle to correctly determine the length of the prosthetic cover. The measurements taken will be further used in creating a size database for correctly subdivide and classy into individual sizes. 


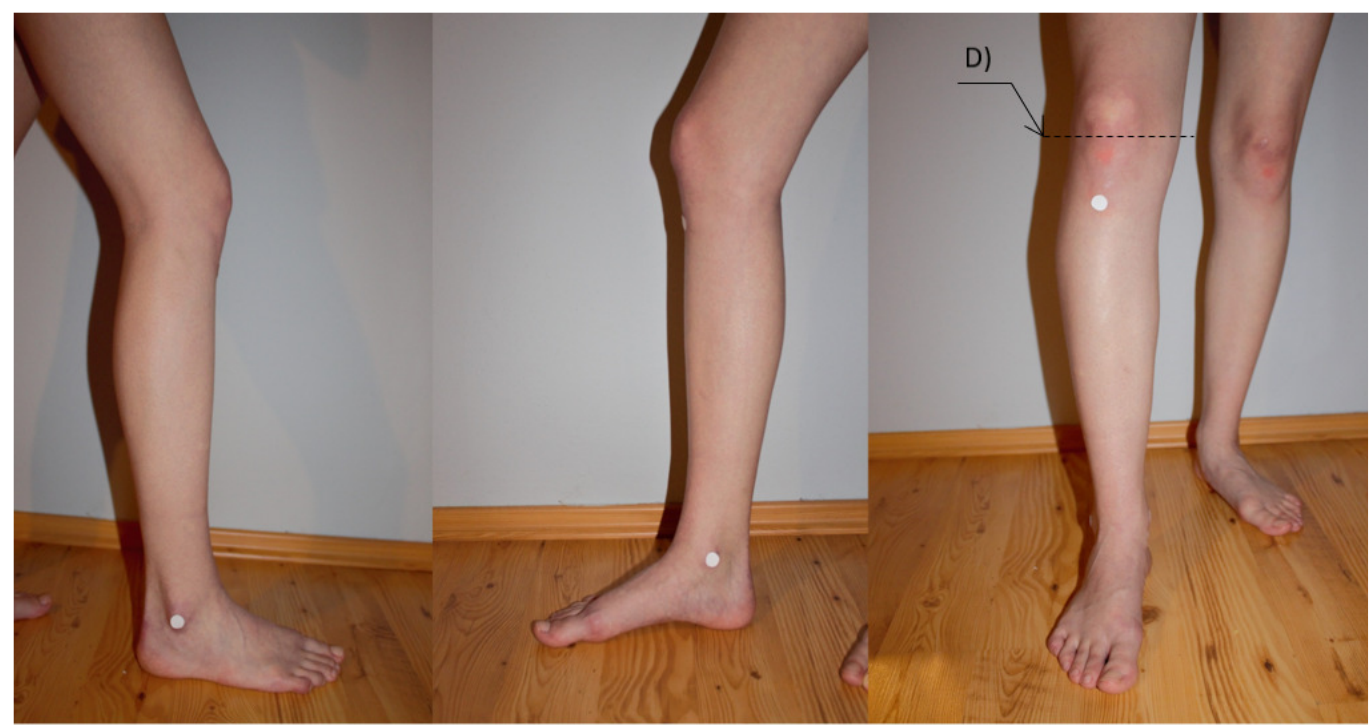

A)
B)
C)

Fig. 1 Anthropometric points: A) the centre of the outer ankle, B) the centre of the inner ankle, $C)$ the roughness of the tibia, D) the axis of the knee joint.

\subsubsection{Manual measurement}

The circumferential and length measurements are chosen so that it is possible to compile a size chart for serial production.

1. Circumferential measurements:

a) circumference below the knee,

b) maximum calf circumference,

c) circumference above the ankles.

2. Length measurements:

a) maximum length from the outer ankle to the axis of the knee joint,

b) height of the maximum calf circumference to the outer ankle.

The manual measurement is performed while the subject is standing, the leg is loaded, exposed and in slight flexion to reveal the muscles that could affect the circumferential measurements. These measurements were chosen because they determine the required length of the prosthetic cover for the pre-knee prosthesis, since this cover extends from the prosthetic foot to the roughness of the tibia. The selected circumferential measures are necessary for the correct shape design and fastening, so that it is correctly adhered to the prosthesis socket, does not stand out unnecessarily and ensures symmetry with the healthy limb.

\subsubsection{D scanning}

Scanning took place under normal temperature conditions $\left(20^{\circ} \mathrm{C}\right)$ so that the subject being scanned did not sweat and at the same time was not cold. Subject's position is standing on both legs, while the scanned limb is loaded, slightly advanced with a slight flexion in the knee joint. The foot is in contact with the pad, and there is a very slight plantar flexion in the ankle joint. Adherence to position is very important, because the scan models will be further used to create size categories and an average model for each size category. It is necessary for the subject to remain in this position for about 3 minutes depending on the scanning speed and quality. This position was chosen mainly due to the use of a 3D scanner, where it is necessary that the limb itself is scanned from all sides $\left(360^{\circ}\right)$. The final output (Fig. 2) for processing in a CAD (computer aided design) software is a model in STL (standard triangle language) format. 


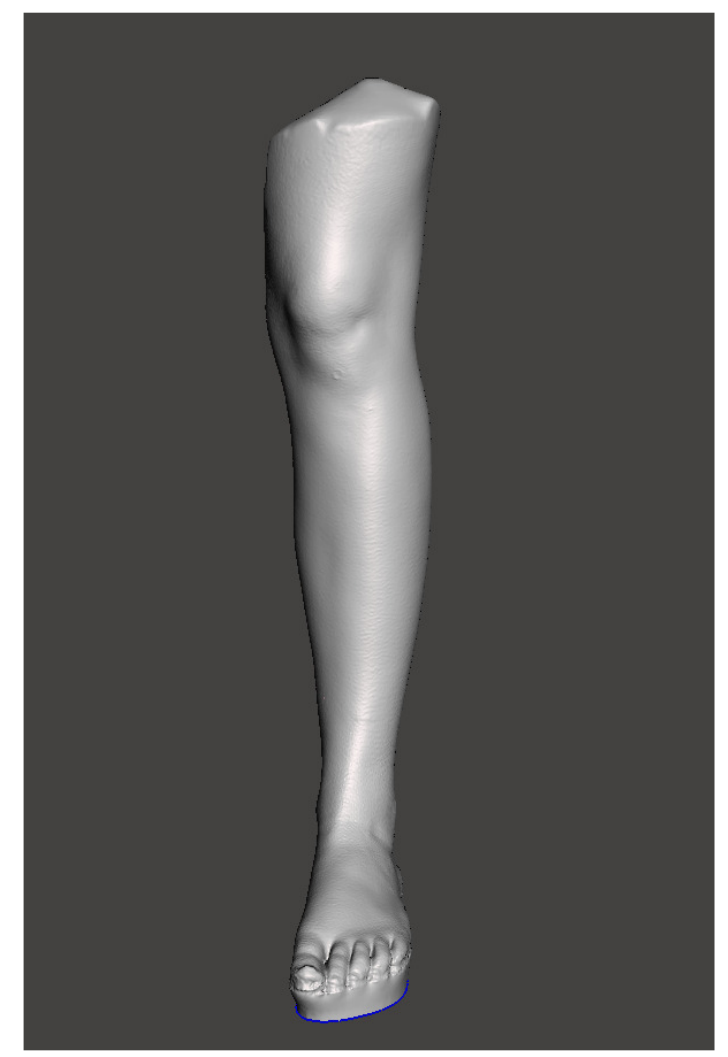

Fig. 2 Scan model in STL format.

\subsubsection{Creation of size categories}

The classification was performed based on measured values, specifically the basic parameter for which the maximum length was chosen from the outer ankle to the axis of the knee joint, because according to this length it is possible to determine the total length of the prosthetic cover. The values of these lengths were arranged in ascending order and then circumferential measurements were assigned to them. This arrangement of measurements was performed and size tables from other companies were also considered in the classification. After creating the database, the individual sizes were divided into $S$ (small), M (medium), L (large) in a certain range (Tab. 1). This range was created based on the respective measured values of both limbs of 17 subjects.

Tab. 1 Size chart.

\begin{tabular}{|c|c|c|c|}
\hline Size & S & M & L \\
\hline $\begin{array}{c}\text { Maximum length from the outer ankle } \\
\text { to the axis of the knee joint [cm] }\end{array}$ & $35-40,99$ & $41-45,99$ & $46-50,99$ \\
\hline Ankle circumference [cm] & $19-21,99$ & $22-24,99$ & $25-27,99$ \\
\hline Maximum calf circumference [cm] & $30-34,99$ & $35-38,99$ & $39-42,99$ \\
\hline Knee circumference [cm] & $29-32,99$ & $33-35,99$ & $36-39,99$ \\
\hline $\begin{array}{c}\text { Height of the maximum circumference } \\
\text { of the calf to the outer ankle [cm] }\end{array}$ & $22-24,99$ & $25-27,99$ & $28-31,99$ \\
\hline
\end{tabular}

\subsection{Average sizes model design}

The perimeter curves of the scans were obtained using VG Studio MAX software (Volume Graphics, Heidelberg, Germany), which allows 3D scans for S, M and L sizes to be overlaid. This scan overlap was chosen to prepare an average model for each size. It was important to cut off unnecessary parts of the scan model above the knee and under the foot, for better alignment. For the functionality of the scans overlap, it was important to align them in two phases. In the first phase, it was necessary to position the limb in space so that the SFTR (sagittal-frontal-transversal) system for orientation in space 
was kept. The plane of the sole of the foot was chosen as the transverse plane and the line formed by a line passing through two points, the inner ankle, and the centre of the big toe as the direction lying in the sagittal plane. As a result, a suitable vertical alignment and consequently the correct rotation of the scans in space has been achieved (Fig. 3). The point on the outer ankle, which determines the beginning of the coordinate system, was chosen as the zero point for the alignment. In the second phase of alignment, the Best-fit function was performed, where a reference scan was selected (average scan based on the median value). Subsequently, according to this scan, other scans were aligned as well.

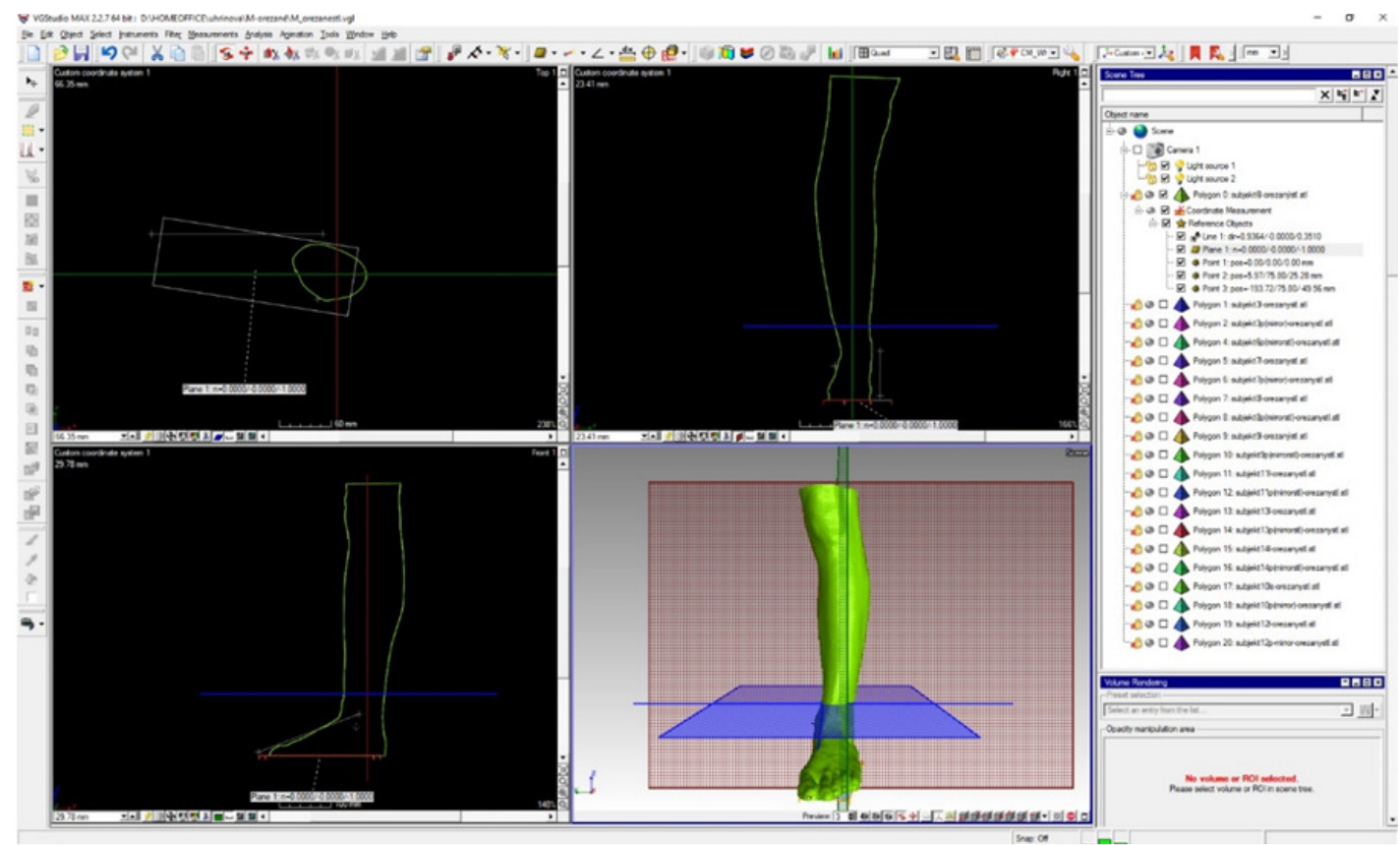

Fig. 3 Average scan model alignment.

After the scans overlap, some scans do not overlap sufficiently even after defining the necessary parameters (failure to maintain the correct position when scanning, greater or smaller abduction or adduction in the lumbar joint, valgus or varus position of the knees and ankles).

By displaying the transverse plane, it is possible to see the circumferential contours of the scans as well as their relative position (

Fig. 4). For the plane below the knee, a height of $340 \mathrm{~mm}$ from the outer ankle was chosen because this height is approximately $20 \mathrm{~mm}$ below the knee. These scans are removed to avoid distortion of the results, which could compromise the resulting model for a given size category. Ultimately, two of all contours were selected, the one with the largest and smallest circumference, to make the average circumference of the model in the section (Fig. 5).

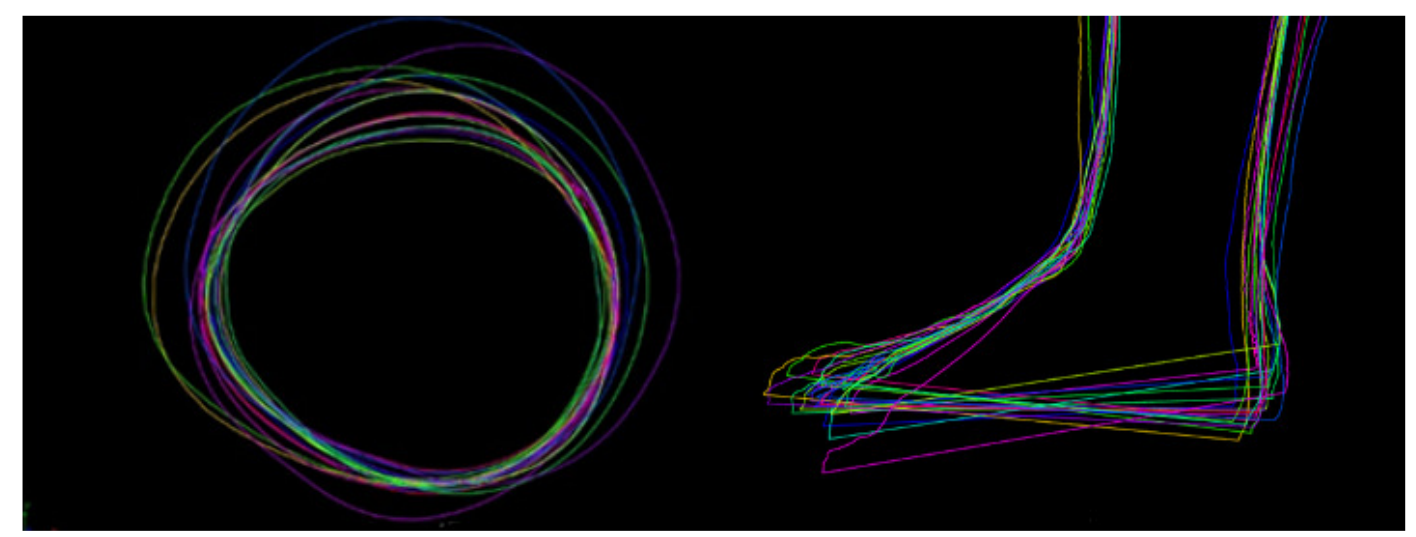

Fig. 4 Scans overlap. 


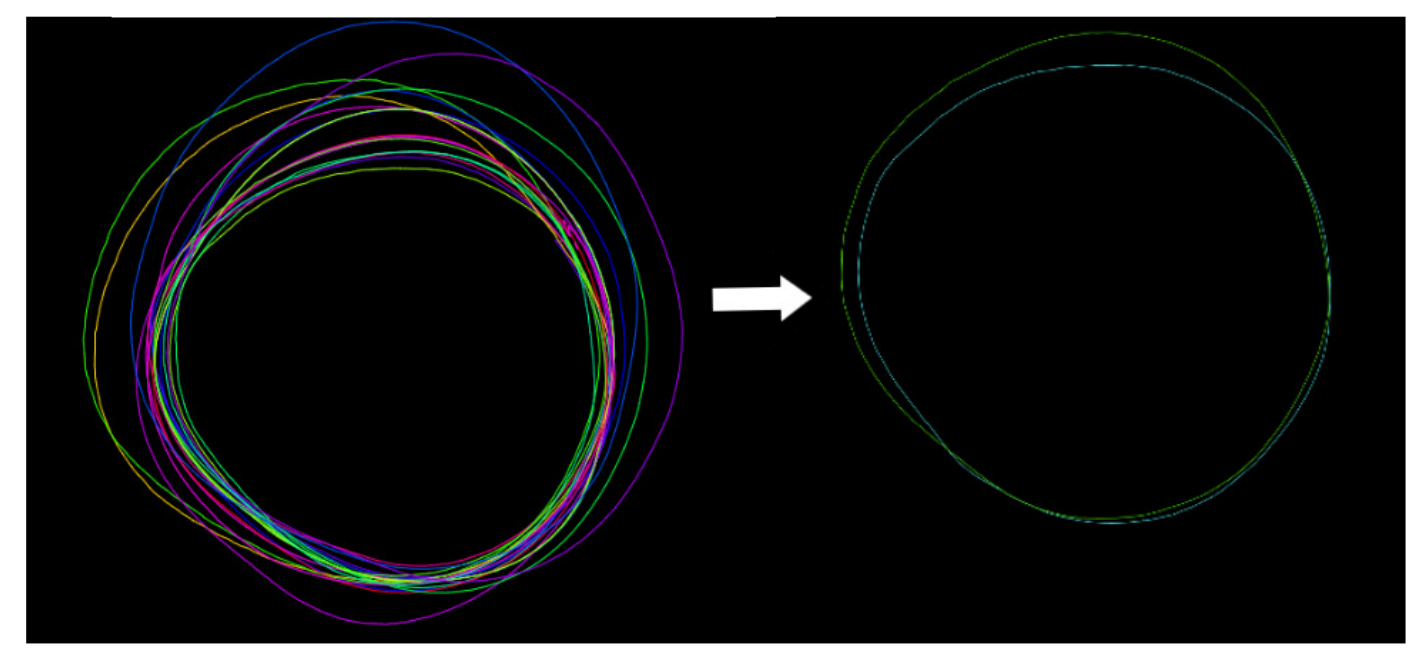

Fig. 5 Display of contours in the transverse plane with subsequent separation of unsuitable contours.

For the average model creation of each size category, 2 circumferential contours were obtained in sections (every $50 \mathrm{~mm}$ of the height), from which average curves were subsequently created in AutoCAD (Autodesk, Inc., San Rafael, CA, USA) software. The average curves were then processed in Fusion 360 (Autodesk, Inc., San Rafael, CA, USA). These curves were distributed at certain distances, as they were cut in the VG Studio MAX software, then connected using the "Loft" function, and the result was an average model for each size category (Fig. 6). These models will serve as a basis for the prosthetic covers design.
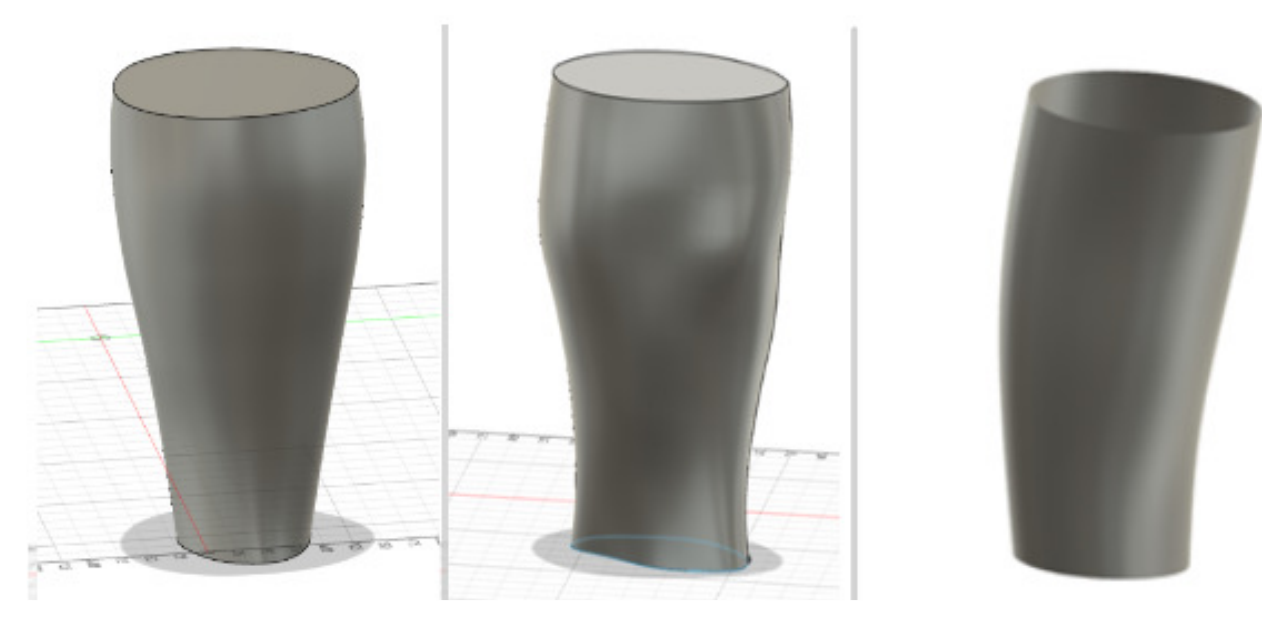

Fig. 6 Average models for size S, $M, L$.

\subsection{Prosthetic covers design process}

The prosthetic cover is designed for transtibial prostheses and consists of 2 parts (front and back), which are connected to each other by means of a pin lock. This basic design was used in all other design modifications of the front part. Several software (Meshmixer, Fusion 360, SolidWorks) were used to develop the structural design and modern look of the prosthetic cover. The main reason was the need to combine nonparametric and parametric modelling.

\subsubsection{Prosthetic cover design}

The original model of the prosthetic cover was designed in Meshmixer (Autodesk, Inc., San Rafael, CA, USA), using a 3D scan. The design consists of 2 parts, the front, and the back, where the back is permanent, because there is a clip for clamping to the prosthesis tube and the front is replaceable (Fig. 7). The main reason for creating a replaceable front part was to offer more options for users and easier adaptation of the prosthesis to everyday life situations. 


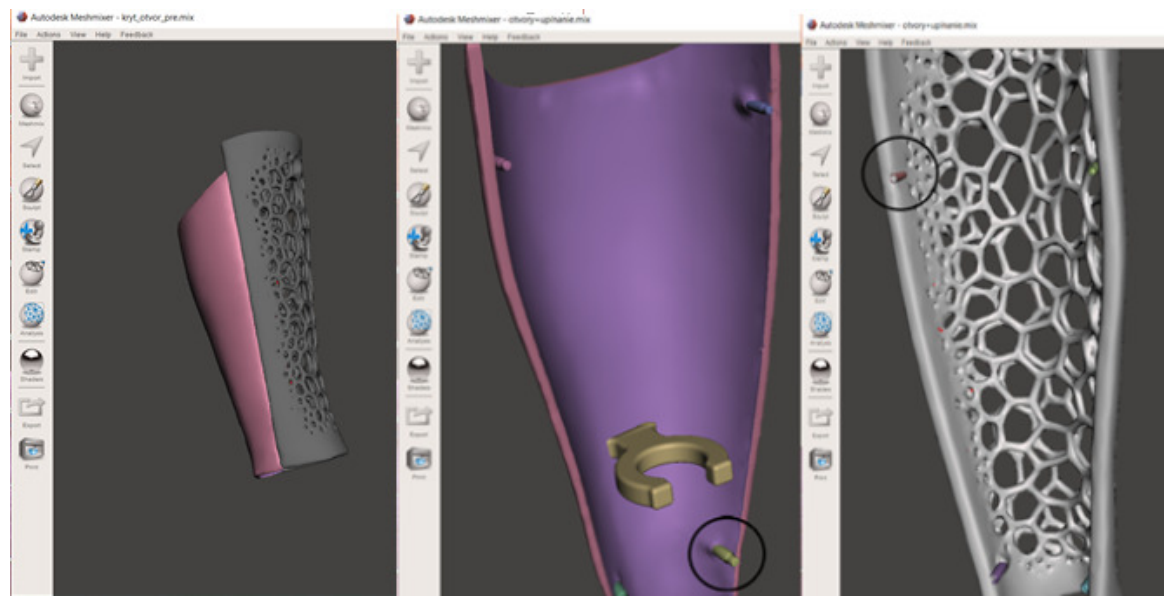

Fig. 7 Prosthetic cover designed in Meshmixer.

The thickness of the prosthetic cover was set to $3 \mathrm{~mm}$ and the connection of the individual parts of the cover is achieved by means of 4 cylindrical pins, which allows the user to an easy access to the prosthesis components as well as easy handling of the cover. Meshmixer is severely limited in the range of design features, and for this reason, other variants of prosthetic covers have been designed in Fusion 360 (Fig. 8). This software was chosen because it allows the creation of surfaces, various designs and working in $2 \mathrm{D}$ and $3 \mathrm{D}$ space. Cover model design was made on the same $3 \mathrm{D}$ scan as when using Meshmixer. The wall thickness of the model was set to $3 \mathrm{~mm}$ and consists of 2 parts, the front, and the back. The mounting of the cover is constructed in the same way as in the previous version and the connection of the individual parts is achieved by means of 4 cylindrical pins.

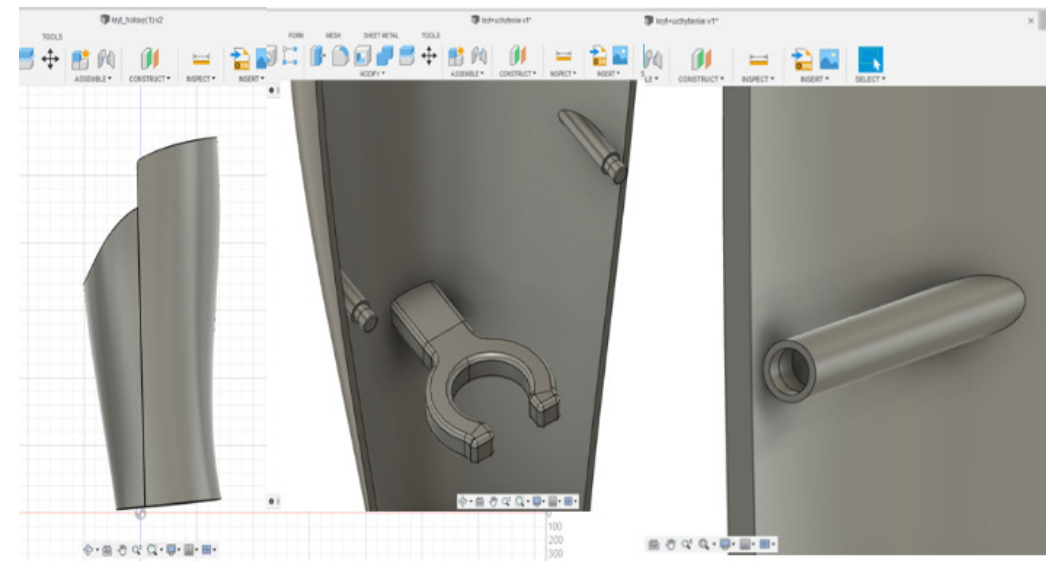

Fig. 8 Prosthetic cover parts designed in Fusion 360.

Three types of covers were designed, which are the "organic" type and 2 other types, which contain various shapes and patterns (Fig. 9).
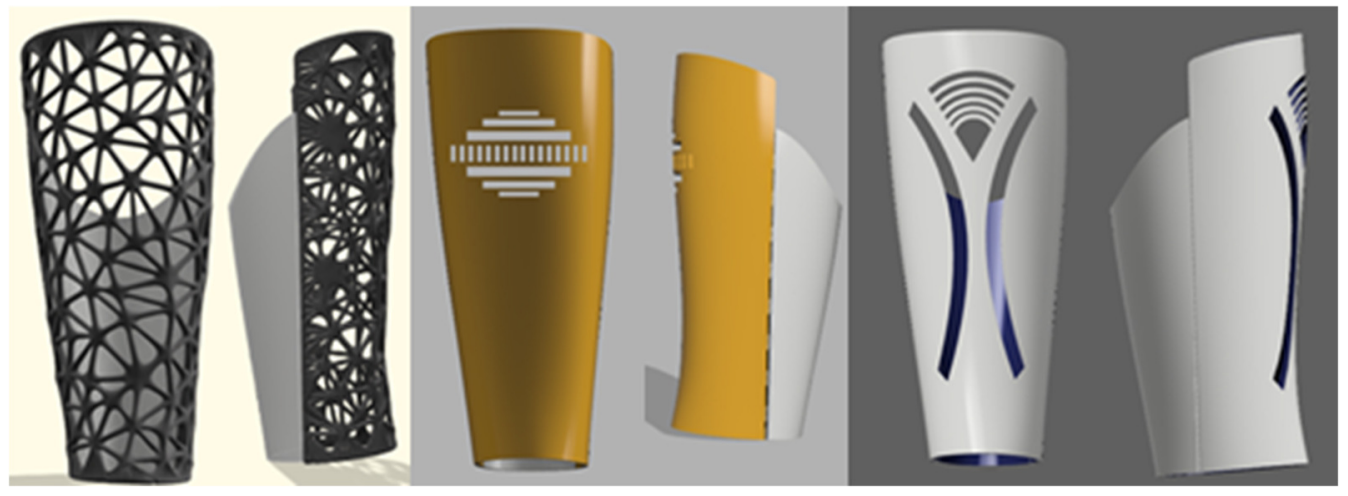

Fig. 9 Prosthetic cover variations. 


\subsection{Manufacturing costs analysis}

It took an average of 2 hours to design the basic prosthetic cover and an average of half an hour to create the custom look, which is ultimately applied to the basic model. The designing time length depends on the patterns, shapes, and technique used in the process. It took an average of 1 hour to model the attachment of the prosthetic cover to the prosthesis itself. The length of manufacturing depends mostly on the size of the model and complexity of the surfaces. The production time was calculated to about 41,4 hours, while the back of the cover was calculated to 12,9 hours and the front to 28,5 hours. $424 \mathrm{~g}$ of material should be consumed for production. The 3D model processing, site preparation and postprocessing has been stated to 3 hours total (Tab. 2).
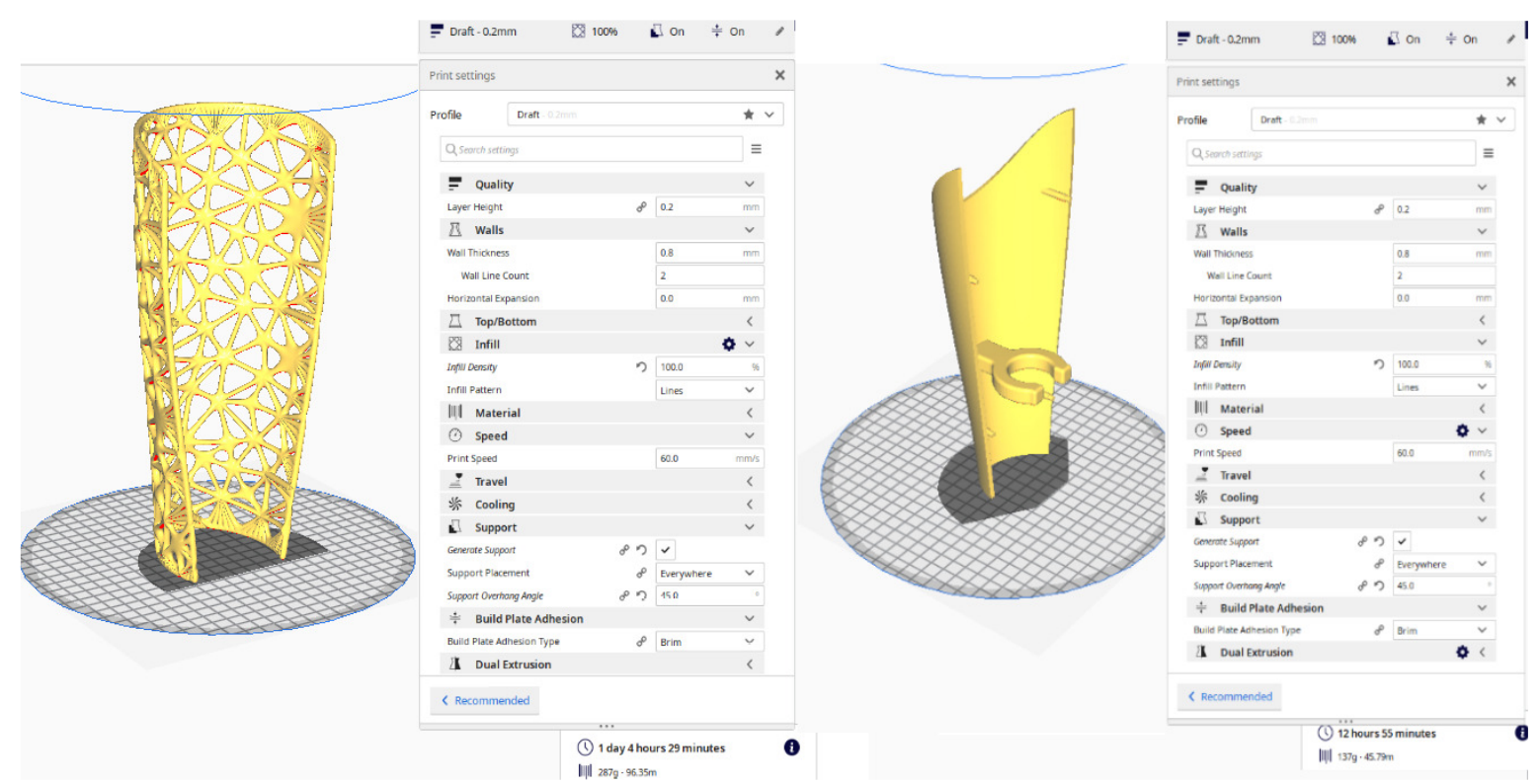

Fig. 10 Virtual 3D model nesting and production parameters setting.

TRILAB DeltiQ 2 FFF (TriLAB Group s.r.o., Brno, Czech Republic) 3D printer with the PLA (polylactic acid) and ABS (acrylonitrile butadiene styrene) material has been chosen for the prototype manufacturing. To calculate the price of the prosthetic cover itself, it was necessary to consider several parameters, namely modelling time in CAD software, preparation for printing, consumed material, printing time and final adjustments. These parameters were given a single price per hour. The price for the work in CAD software was set at $50 € /$ hour, the preparation of the print itself was given at $3 € /$ hour, printing on FFF printer was set to $2 € /$ hour and final adjustments were charged at $3 € /$ hour (Tab. 2). The cost of the amount of material was calculated based on the material consumed in kilograms (Tab. 3).

Tab. 2 Manufacturing costs calculation of 1 prosthetic cover.

\begin{tabular}{|c|c|c|c|}
\hline Parameter & Hours & $\begin{array}{c}\text { Cost per hour } \\
(\boldsymbol{\epsilon} / \text { hour })\end{array}$ & Price (€) \\
\hline Basic design & 2 & 50 & 100 \\
\hline Mounting design & 1 & 50 & 50 \\
\hline Custom look design & 1 & 50 & 50 \\
\hline Model processing & 1 & 3 & 3 \\
\hline Additive manufacturing & 41,4 & 2 & 82,80 \\
\hline Postprocessing & 2 & 3 & 6 \\
\hline Total cost & & & $\mathbf{2 9 1 , 8 0}$ \\
\hline
\end{tabular}

Tab. 3 Material costs calculation of 1 prosthetic cover.

\begin{tabular}{|c|c|c|c|}
\hline Material & $\begin{array}{c}\text { Amount of material } \\
\text { used }(\mathbf{k g})\end{array}$ & Price of material $(\boldsymbol{\epsilon} / \mathbf{k g})$ & Total cost \\
\hline PLA & 0,424 & 20,70 & $\mathbf{8 , 7 8}$ \\
\hline ABS & 0,424 & 23,40 & $\mathbf{9 , 9 2}$ \\
\hline
\end{tabular}


Based on the calculation above, after considering a $10 \%$ surcharge, a sum of $330,63 €$ was calculated for a prototype cover made of PLA material and $331,89 €$ for the prototype cover made of ABS material (Tab. 4). Since the aim was to design a prosthetic cover, which will be mass-produced, a variant of 10 pieces and 100 pieces was calculated. The total amount may vary for individual proposed variants due to the choice of a different type of 3D printer and different setting of 3D printing parameters. The resulting cost can also be slightly changed depending on the amount of material used for each type of prosthetic cover.

When the prosthetic cover was duplicated into 10 pieces, the design price for one piece was calculated to the amount of $20,30 €$, the material amount of $8,78 €$ for PLA material and 9,92€ for ABS material, 3D printing of $82,80 €$ and $6 €$ surface treatment has been added. The total amount for one cover of the 10piece serial production is $117,88 €$ for PLA and 119,02 for ABS material. Considering the $10 \%$ surcharge, the final amount for one PLA cover is $129,66 €$ and $130,92 €$ for one ABS cover (Tab. 4).

When the prosthetic cover was duplicated into 100 pieces, the design price for one piece was calculated to the amount of $2,03 €$, the material amount of $8,78 €$ for PLA material and $9,92 €$ for ABS material, 3D printing of $82,80 €$ and $6 €$ surface treatment has been added. The total amount for one cover of the 100 piece serial production is $99,61 €$ for PLA material and $100,75 €$ for ABS material. Considering the $10 \%$ surcharge, the final amount for one PLA cover is $109,57 €$ and $110,83 €$ for one ABS cover (Tab. 4).

Tab. 4 Final production costs calculation.

\begin{tabular}{|c|c|c|c|}
\hline Production & 1-piece & 10 -piece & 100-piece \\
\hline Final cost per PLA piece $(\boldsymbol{\epsilon})$ & 330,63 & 129,66 & 109,57 \\
\hline Final cost per ABS piece $(\boldsymbol{\epsilon})$ & 331,89 & 130,92 & 110,83 \\
\hline
\end{tabular}

\section{Results and discussion}

The foreleg measurements for the custom cover design were executed in two phases, using a manual method and 3D scanning, where 3D scanning appeared to be more efficient, faster, and precise. Measurements were taken from 17 subjects, which is not enough for such a purpose, but due to the pandemic situation, it was not possible to collect measurements from more subjects. In the future, it is recommended to measure more subjects for the purpose of creating a more relevant average prosthetic cover. However, when 3D scanning, it is important for the subject to remain in a certain position for a few minutes and it must be adhered during each scan. Failure to keep this position can caused incorrect scans, which may affect the results in the designing process. To maintain this position correctly, it is appropriate to mark places on the ground for the feet and a use at least two lasers (to check compliance), so the scanned subjects maintain in one place.

When creating a database for serial production, it is necessary to select the main parameter, from which the classification of measured values will be derived, in this case it was the maximum length from the outer ankle to the axis of the knee joint. After dividing the scans into individual size categories, this parameter appeared to be insufficient, because some scans significantly outperformed others, which could be caused by non-compliance with the scanning position or incorrect classification of measured values. For a more detailed and accurate distribution of the measured values, it would be more efficient to choose not only the length measure as the main parameter, but also one circumferential, such as the circumference below the knee, which could specify the result.

To create an average model for each size category, average curves were made at certain distances $(50 \mathrm{~mm})$, which initially seemed sufficient, but when creating the average model itself, it was found that it is necessary to make a thicker cut, resulting in a much more accurate model. This distance caused a slight distortion of the resulting model. For the design process, 3 brands of CAD software were chosen (SolidWorks, Meshmixer, Fusion 360), where in the first phase, Meshmixer appeared to be a suitable software, but when creating the 3D model of the cover it was considerably limited. For this reason, Fusion 360 and SolidWorks were used, offering a wider range of design features and functions.

In this work, 3 specific types of the resulting models of prosthetic covers were proposed, for which a calculation was made in the final phase. The Organic prosthetic cover was selected for production serial production calculation on a TRILAB Deltiq 2 FFF 3D printer. From the results of the calculation, it can be stated, that there is no significant difference between the total manufacturing costs when using PLA or ABS material. Another result is that a serial production of custom prosthetic covers on a FFF 3D printer is more cost efficient if there is a greater amount (10-100 pieces) of covers for production. 
When we compare the price of the proposed prosthetic cover (around 330 euro a piece) to the price of commercially available 3D printed cover, of which average value is approximately 800 euros, we have a difference of approximately 470 euros. This means, that the proposed custom prosthetic cover can financially compete with products of commercial companies on the market [8-11].

\section{Conclusions}

The presented work confirms the suitability of $A M$ as an option for the mass production of custom prosthetic covers, also the financial analysis is favourable in comparison with commercially available products, and it offers the estimated cost of the cover's serial production. The current possibilities of using innovative technologies offer many advantages for simplification, acceleration, but first and foremost for the personalization of prosthetic and orthotic devices, which makes them an ideal solution either in data obtainment or in the production itself. The disadvantages associated with new technologies may be higher initial equipment costs and the need for a certified professional staff, but in the current trend it is only a small investment in the future competitiveness capabilities.

Future works include low-cost additive manufacturing, and mechanical and practical testing of the proposed prosthetic cover. It would be also relevant to compare manufacturing costs with a high-end 3D printer, suitable for serial production.

\section{Acknowledgements}

This work was supported by the Slovak Research and Development Agency under the contract No. APVV-19-0290, project KEGA 041TUKE-4/2019, and KEGA 023TUKE-4/2020. The achieved results were created within the investigation of the project no. 2018/14432: 1-26C0, which is supported by the Ministry of Education, Science, Research and Sport of the Slovak Republic within the provided incentives for research and development from the state budget in accordance with Act No. 185/2009 Coll. on incentives for research and development.

\section{References}

[1] Facts about limb loss, https://www.sralab.org/research/labs/max-nader-center-rehabilitation-technologies-and-outcomes-research/news/facts-about-limb-loss., accessed 2021.

[2] C.-A. Behrendt et al., "International Variations in Amputation Practice: A VASCUNET Report," doi: 10.1016/j.ejvs.2018.04.017.

[3] Dochází u nás k poklesu amputací dolních končetin?, http://www.address.cz/data/www.sanquis.cz/articles/files/62_dochazi_k_amputacim_dolnich_koncetin.pdf., accessed 2021.

[4] CD. Murray, and J. Fox, "Body image and prosthesis satisfaction in the lower limb amputee," Disabil. Rehabil., vol. 24, no. 17, pp. 925-931, Nov. 2002, doi: 10.1080/09638280210150014.

[5] Amputee Coalition of America (ACA): PeopleWith Amputation SpeakOut. The Limb Loss Research and Statistics Program Johns Hopkins Bloomberg School of Public Health, https://www.amputee-coalition.org/wp-content/uploads/2014/11/lsp people-speak-out 120115113243.pdf., accessed 2021.

[6] S. Sansoni, L. Speer, A. Wodehouse, and A. Buis, "Aesthetic of Prosthetic Devices: From Medical Equipment to a Work of Design," Emot. Eng. Vol. 4, pp. 73-92, Jan. 2016, doi: 10.1007/978-3319-29433-9_5.

[7] S. Ritchie, S. Wiggins, and A. Sanford, "Perceptions of cosmesis and function in adults with upper limb prostheses: A systematic literature review," Prosthet. Orthot. Int., vol. 35, no. 4, pp. 332-341, Dec. 2011, doi: 10.1177/0309364611420326.

[8] LIMB-art Cool Prosthetic Leg Covers, https://limb-art.com/., accessed 2021.

[9] Art4leg, https://art4leg.com/., accessed 2021.

[10] Below-Knee Cover, https://alleles.ca/order/bk-cover/., accessed 2021

[11] Prosthetic covers designs, https://unyq.com/en-us/prosthetic-covers/designs-us/., accessed 2021 